1) Title page

a) Full title

A novel locally operated master-slave robot system for single-incision laparoscopic surgery

b) Running title

A novel locally operated master-slave robot system for single-incision laparoscopic surgery

c) All contributing authors with full name, title and affiliation

Yuki Horise, Ms.,

Department of Mechanical Science and Bioengineering, Graduate School of Engineering Science, Osaka University, Toyonaka, Japan

Toshinobu Matsumoto, Mr.,

Department of Robotics, Faculty of Engineering, Osaka Institute of Technology, Osaka, Japan

Hiroki Ikeda, Mr.,

Department of Robotics, Faculty of Engineering, Osaka Institute of Technology, Osaka, Japan

Yuta Nakamura, Mr.,

Department of Robotics, Faculty of Engineering, Osaka Institute of Technology, Osaka, Japan

Makoto Yamasaki, Assistant Prof., Dr.,

Department of Gastroenterological Surgery, Graduate School of Medicine, Osaka University, Suita, Japan

Genta Sawada, Dr.,

Department of Gastroenterological Surgery, Graduate School of Medicine, Osaka University, Suita, Japan

Yukiko Tsukao, Dr.,

Department of Gastroenterological Surgery, Graduate School of Medicine, Osaka University, Suita, Japan

Yujiro Nakahara, Dr.,

Department of Gastroenterological Surgery, Graduate School of Medicine, Osaka University, Suita, Japan

Masaaki Yamamoto, Dr.,

Department of Gastroenterological Surgery, Graduate School of Medicine, Osaka 
University, Suita, Japan

Shuji Takiguchi, Associate Prof., Dr.,

Department of Gastroenterological Surgery, Graduate School of Medicine, Osaka University, Suita, Japan

Yuichiro Doki, Prof., Dr.,

Department of Gastroenterological Surgery, Graduate School of Medicine, Osaka University, Suita, Japan

Masaki Mori, Prof., Dr.,

Department of Gastroenterological Surgery, Graduate School of Medicine, Osaka University, Suita, Japan

Fumio Miyazaki, Prof.,

Department of Mechanical Science and Bioengineering, Graduate School of Engineering Science, Osaka University, Toyonaka, Japan

Mitsugu Sekimoto, Dr.,

Osaka National Hospital, Osaka, Japan

Toshikazu Kawai, Associate Prof.,

Department of Robotics, Faculty of Engineering, Osaka Institute of Technology, Osaka, Japan

Atsushi Nishikawa, Prof.,

Division of Mechanical Engineering and Robotics, Faculty of Textile Science and Technology, Shinshu University, Ueda, Japan

d) Corresponding author details

Atsushi Nishikawa, Prof.,

Division of Mechanical Engineering and Robotics, Faculty of Textile Science and Technology, Shinshu University, Ueda, Japan

3-15-1 Tokida, Ueda, Nagano, 386-8567, Japan

$+81-268-21-5617$

nishikawa@shinshu-u.ac.jp 


\section{2) Abstract}

\section{Purpose}

Single-incision laparoscopic surgery (SILS) provides more cosmetic benefits than conventional laparoscopic surgery but presents operational difficulties. To overcome this technical problem, we have developed a locally operated master-slave robot system that provides operability and a visual field similar to conventional laparoscopic surgery.

\section{Methods}

A surgeon grasps the master device with the left hand, which is placed above an abdominal wall, and holds a normal instrument with the right hand. A laparoscope, a slave robot, and the right-sided instrument are inserted through one incision. The slave robot is bent in the body cavity and its length, pose, and tip angle are changed by manipulating the master device; thus the surgeon has almost the same operability as with normal laparoscopic surgery.

To evaluate our proposed system, we conducted a basic task and an ex vivo experiment.

\section{Results}

In basic task experiments, the average object-passing task time was $9.50 \mathrm{sec}$ (SILS cross), $22.25 \mathrm{sec}$ (SILS parallel), and $7.23 \mathrm{sec}$ (Proposed SILS). The average number of instrument collisions was 3.67 (SILS cross), 14 (SILS parallel), and 0.33 (Proposed SILS). In the ex vivo experiment, we confirmed the applicability of our system for single-port laparoscopic cholecystectomy.

\section{Conclusion}

We demonstrated that our proposed robot system is useful for single-incision laparoscopic surgery.

3) Keywords

Local operation, Master-slave, Laparoscopic surgery, Single incision 
4) Main text

a) Introduction

Laparoscopic surgery in which surgical instruments and a laparoscope are put through multiple incisions has been used for various operations because of its numerous benefits like smaller scars, less pain, and shorter hospitalization than open surgery. To reduce the number of scars and lessen scar pain, single-incision laparoscopic surgery (SILS), which requires only a single incision, has been developed. This method is more cosmetic than laparoscopic surgery and is called "scarless surgery" since the scar is hidden if the incision is made at an umbilicus (1, 2). However, SILS presents technical problems due to its single opening, such as a narrow area, internal and external interference between the instruments, and field-of-view issues. SILS is thus more difficult than multi-incision surgery, and the surgeons' burden is increased (3-5). Equipment for ports and instruments has been improved over the last 7 years, and the constraints in ergonomics of and technologies for SILS have been partially resolved (6).

Recently, several surgical robot systems have been developed to address the problems in SILS, and the da Vinci Surgical System (Intuitive Surgical Inc.) is an especially good example. The da Vinci system has been used worldwide in many fields such as urology, gastroenterology, and gynecology (7-13) because this robot offers a 3D visual field, precise manipulation, and hand-movement cancellation (14). Although the da Vinci is becoming a common surgical robot, there are some problems: collisions of arm robots, lack of space, a lengthy setup time, and high cost for introduction and maintenance (15-17). Moreover, it takes a long time for skilled surgeons to learn the operation of the robot (18).

As an alternative, the bimanual robot system (SPRINT surgical platform) proposed by Piccigallo et al. (19) from Pisa University is a master-slave platform designed for bimanual interventions through a single port. SPRINT is assembled in vivo and surgeons are able to control a slave robot in a master-slave configuration through a dedicated console. Instrument collisions outside of the body like those that occur with the da Vinci system do not occur because the two slave arms move inside the body cavity. In the master-slave surgical robot system for SILS, developed by Kobayashi et al. from Waseda University (20), surgical instruments and a camera are gathered together in one pipe. Surgeons can resect the tissues without instrument collisions by controlling its master manipulators. Our research group also has developed a master-slave robot system with which surgeons can conduct SILS as if they were performing conventional laparoscopic (multi-incision) surgery (21). This 
system provides surgeons more efficient and intuitive operation than does conventional SILS, although it does have problems such as instrument collisions. In these robot systems, the operator uses a PHANTOM or dedicated device to control the slave robot, and so surgeons have to learn these manipulations from scratch. The robot systems also have the potential to injure the body due to machine trouble because in remote operation the surgeons do not stand at the side of the patient and cannot touch the tissues directly. In particular, SPRINT has a risk of electric shock since DC motors mounted in the slave arms are used inside the body cavity. The surgical robotic manipulator designed by Natali et al. from Vanderbilt University (22) employs internal and external magnets to control robotic arms inside the cavity, which were inserted via a single incision through an abdominal wall. However, this robotic manipulator could cause internal bleeding of the abdominal wall and is not minimally invasive.

In order to overcome the problems in SILS using an approach differing from the above studies, we proposed and developed a locally operated master-slave robot system which provides operability and a visual field similar to conventional laparoscopic surgery. We evaluated our proposed system by conducting a basic object-moving task and an ex vivo experiment using a porcine liver.

b) Material and Methods

Locally operated master-slave robot system

- System overview

As seen in Figure 1 (a), our proposed system is configured with a master device, a slave robot, and a control PC. A surgeon uses the master device, which is placed "above" an abdominal wall of the patient and typically set at an appropriate position using the conventional passive holder mounted on the surgical table. The slave robot, which is controlled by the master device, is inserted from a single incision and set under the master device through the abdominal wall.

When using our system to perform SILS, the operating surgeon holds a commercial forceps with the right hand while manipulating the master device with the left hand. A laparoscope, the right hand forceps, and the slave robot are all put through one incision. The length, pose, and tip opening and closing of the "slave" robotic instrument can be controlled by manipulating the "master" input instrument. These two instruments thus behave as if they were one normal instrument. 
(a)

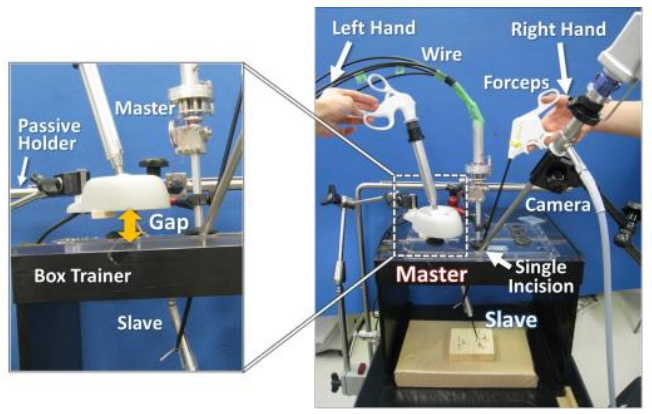

(b)

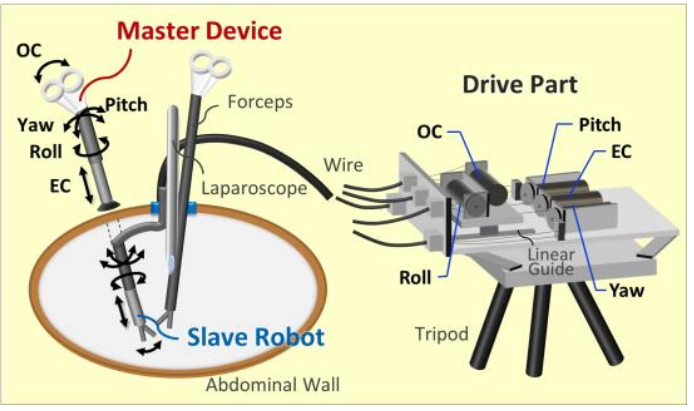

Fig. 1

Novel locally operated master-slave robot system for SILS. (a) Real robot system.

(b) System concept.

\section{- Master device}

The master device, as illustrated in Figure 1 (b), has five DOF motion, the same as the usual forceps, specifically extension and contraction (EC) along the longitudinal axis, spherical motion (Yaw and Pitch) around the set point, rotation (Roll) around the longitudinal axis, and grip opening and closing (OC). These motions are measured in real-time with the following five sensors equipped with the master device: a rotary potentiometer, a linear potentiometer, an acceleration sensor, a gyro sensor, and a geomagnetic sensor. Notice, however, that the master device does NOT pass through the abdominal wall, which means that there are gaps between them (see Figure 1 (a)).

\section{- Slave robot}

The slave robot has the same five DOF as the master device achieved by using a wire-driven mechanism with five external DC motors (Figures 1 (b) and 2 (a)) and consists of a passive arm and an active robotic instrument. The tip of the arm is fixed to a passive holding arm placed across the operating table. The slave arm has an articulated mechanism in which a couple of articulations bend up to 90 degrees, and can convert from straight to bent when the wire is pulled as indicated in Figure 
2 (b); it is straight when passing through the port and bent when in operation. The other end of the arm is connected to the robotic instrument through two silicon rubber plates that are supposed to achieve 60 degrees of bending angle. In the slave performance experiment with the arm bent, the $10 \mathrm{~mm}$-diameter instrument extended and contracted $36.5 \mathrm{~mm}$ and bent 28 degrees in the pitch direction and 20 degrees in the yaw direction. It rotated \pm 180 degrees around the axis and opened and closed continuously. The positioning accuracy of the instrument was $1.1 \mathrm{~mm}$ in the pitch direction and $1.01 \mathrm{~mm}$ in the yaw direction.

(a)

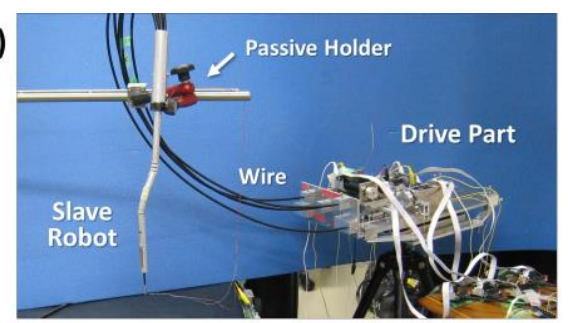

(b)

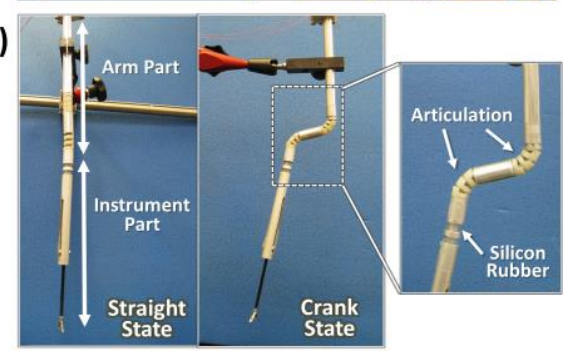

Fig. 2

Flexible slave robot. (a) Slave overview. (b) State changes of the robot.

Evaluation of the locally operated master-slave robot system

- Basic object-moving task

As a basic task to indicate the feasibility of our system, we conducted an object-moving task (23) for three surgeons (Surgeons A, B, and C). Our purpose in this study is to perform SILS more easily, thus we prepared the following three operating conditions: (I) conventional SILS with the instruments crossed, (II) conventional SILS with the instruments parallel, and (III) the proposed SILS using our robot system (Figure 3 (a)). There are the usual two straight forceps in operating conditions (I) and (II), both instruments are inserted into a single port and crossed in operating condition (I); two instruments pass through one port and are used in parallel in operating condition (II). In operating condition (III), we use our robot system as the left-side instrument and normal forceps as the right-side instrument.

There is a peg board in front of the single incision in a box trainer, and three circular 
objects are placed on the left-side pegs of the board (Figure 3 (b)). An operator must lift each object from the left pegs with the left instrument in the laparoscopic view, transfer it to the right side instrument in the laparoscopic view, and place it on a right-side peg. The operator moves a series of objects three times in total for one trial. We recorded the switching time and number of collisions, and compared them among the three conditions. We defined the starting time of the object passing as the time when the right-side instrument approaches the object and opens its tip while the left-side instrument is grasping the object, and the ending time as the time when the left-side instrument releases the object while the right-side instrument is catching the object. Three surgeons conducted three trials each under the three operating conditions and subjectively evaluated each case. Their general experience, including surgical experience in all cases, in laparoscopic surgery, and in single-incision laparoscopic surgery, of the three surgeons is presented in Table 1.

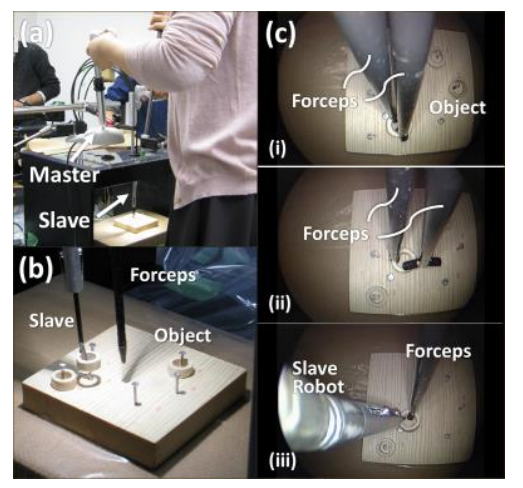

Fig. 3

Setup for the object-moving task. (a) Experiment overview. (b) Task board. (c) Laparoscopic views under three operating conditions: (i) conventional SILS (cross), (ii) conventional SILS (parallel), and (iii) proposed SILS.

Table 1

Surgical experience of surgeons

\begin{tabular}{lcccc}
\hline $\begin{array}{c}\text { Surgeon } \\
\text { ID }\end{array}$ & $\begin{array}{c}\text { Experience } \\
\text { (years) }\end{array}$ & $\begin{array}{c}\text { Experience } \\
\text { (number of } \\
\text { cases) }\end{array}$ & $\begin{array}{c}\text { Experience in } \\
\text { laparoscopic surgery } \\
\text { (number of cases) }\end{array}$ & $\begin{array}{c}\text { Experience in } \\
\text { SILS (number of } \\
\text { cases) }\end{array}$ \\
\hline A & 6 & 582 & 100 & 2 \\
B & 7 & 255 & 50 & 5 \\
C & 8 & 450 & 30 & 0 \\
\hline
\end{tabular}


- Ex vivo experiment using a porcine liver

In this study, we consider that cholecystectomy, which is mostly conducted by using the multi-port method, is one of the surgeries our proposed system targets. Hence, we confirmed the validity of our system for single-port laparoscopic cholecystectomies by conducting an ex vivo experiment using a porcine liver. At the start of the experiment, the master device, the slave robot, and the laparoscope were set on the surgical table. A porcine liver with a gallbladder was fixed on a board in the box trainer, and the cystic duct was clipped in advance as indicated in Figure 4 (a). The master device was placed slightly above the box. The operator manipulated the master device with the left hand and normal resection forceps with the right hand while watching the laparoscopic view on a monitor, and separated the gallbladder from the liver (Figure 4 (b)). The slave robot, the right-hand instrument, and the laparoscope were all put through one incision. The operator provided a subjective evaluation after this experiment.

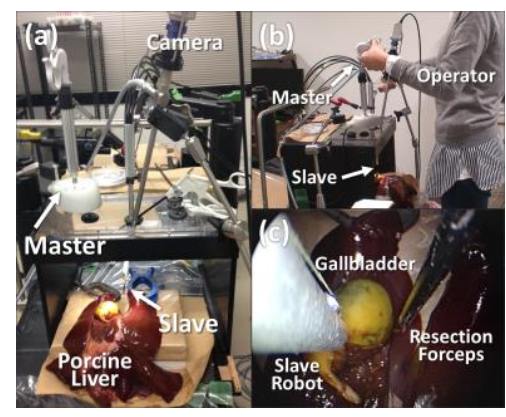

Fig. 4

Ex vivo cholecystectomy using our proposed system. (a) System setup. (b) Side view of master-slave system. (c) Laparoscopic view of the slave robot grasping the gallbladder.

c) Results and Discussion

Results

- Basic object-moving tasks

The average switching time and number of instrument collisions in the object-moving task for each surgeon under the three operation conditions are indicated in Figures 5 (a) and (b).

The average switching time and standard deviation for each operating condition of Surgeon A were $11.65 \pm 14.97 \mathrm{sec}$ (SILS cross), $21.64 \pm 33.85 \mathrm{sec}$ (SILS parallel), and $7.03 \pm 2.49 \mathrm{sec}$ (Robot); those of Surgeon B were $6.82 \pm 4.51 \mathrm{sec}$ (SILS cross), 
$13.82 \pm 29.40 \mathrm{sec}$ (SILS parallel), and $8.48 \pm 3.58 \mathrm{sec}$ (Robot); and those of Surgeon $\mathrm{C}$ were $10.04 \pm 5.07 \mathrm{sec}$ (SILS cross), $35.82 \pm 34.66 \mathrm{sec}$ (SILS parallel), and $6.48 \pm$ $2.25 \mathrm{sec}$ (Robot). The number of collisions for each operating condition of Surgeon A was 7 (SILS cross), 15 (SILS parallel), and 0 (Robot); those of Surgeon B were 3 (SILS cross), 9 (SILS parallel), and 1 (Robot); and those of Surgeon C were 1 (SILS cross), 18 (SILS parallel), and 0 (Robot).

From these data, the average switching time and standard deviation of all surgeons in the three operating conditions were $9.50 \pm 9.70 \mathrm{sec}$ (SILS cross), $22.25 \pm 33.57$ sec (SILS parallel), and $7.23 \pm 2.87 \mathrm{sec}$ (Robot). The average number of collisions between the instruments in the box during the task of all surgeons was 3.67 (SILS cross), 14 (SILS parallel), and 0.33 (Robot). The instrument and the laparoscope collided outside of the box all the time under conventional SILS operating conditions, although the master device did not conflict with the laparoscope or the other instrument when our proposed robot system was used. Laparoscopic views under the three operating conditions are presented in Figure 3 (c).

- Ex vivo experiment using a porcine liver

The operator separated the gallbladder from the liver while grasping it using the slave robot by controlling the master device with the left hand and manipulating the resection forceps with the right hand (Figure 4 (c)). There was no interference between the instruments as in the case with the basic task experiment.

(a)

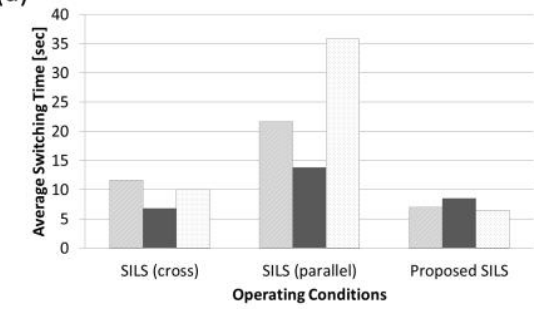

(b)

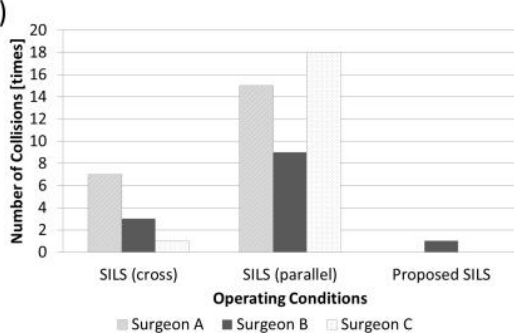

Fig. 5

Results of the object-moving task for three surgeons under three operating conditions. (a) Average switching time. (b) Number of instrument collisions. 


\section{Discussion}

In the results of the object-moving task, the switching time for SILS using our robot system was shorter than that for conventional SILS operating conditions, most notably with the instruments in a parallel state. The same is true for the variation in the task time. We consider that this was due to a shift to the left of the pivot position of the left-side robotic instrument in the proposed operating condition while the forceps for conventional SILS operations were close to each other (Figures 3 (c)-(i), (ii)). Thus, there were fewer collisions between instruments in the operating condition using the robot system than under the other conditions, and the surgeons could operate the system stably. Furthermore, the laparoscopic view in the proposed SILS was similar to that of usual laparoscopic surgery because the robotic instrument approached from the left side as seen in Figure 3 (c)-(iii), whereas the two instruments approached from the same direction in conventional SILS operations; it was unlike other SILS views since the location relationship of the two instruments differed. For this reason, we considered that our proposed SILS had fewer instrument collisions. The surgeons felt more stress in the normal SILS operations since instruments collided often; they felt little stress in the proposed operating condition. They thought that the system had adequate tracking performance and operability and that the laparoscopic view in the proposed SILS was close to that in normal multi-incision surgery. The subjective evaluations indicated that the surgeons' burden was drastically reduced by using our robot system compared to conventional SILS.

In the ex vivo experiment, the slave robot grasped and pulled the gallbladder without any problems like collisions between the instruments. This demonstrated that our robot system had a sufficient workspace and grasping force to perform single-port laparoscope cholecystectomies. As indicated in Figure 4 (c), we found that our robot system had a laparoscopic view similar to multi-port surgery and would provide a surgical environment close to that of normal laparoscopic surgery. A subjective evaluation revealed that the operator conducted the procedure with less stress and more easily than with conventional SILS. The operator also felt that the laparoscopic view was similar to that in usual laparoscopic surgery and that it felt like performing laparoscopic surgery even though there was only one port.

Therefore, surgeons using our proposed master-slave robot system could conduct "single-incision" laparoscopic surgery more comfortably, efficiently, and stably with fewer collisions while observing almost the same laparoscope view as conventional 
"multi-incision" surgery.

These evaluation results mean that our proposed system provides the surgeon a feeling of maneuvering like that with a normal instrument inserted through another virtual incision (i.e., as if there were two ports) although physically there is only one port in the abdominal wall. Moreover, the surgeon has a similar field of view as when performing normal laparoscopic surgery since the robotic arm is cranked and the robotic instrument is straight, not flexed. Therefore, our proposed system provides surgeons operability similar to conventional laparoscopic surgery to make maximum use of their laparoscopic techniques and the decreased number of instrument collisions compared with regular SILS.

In our proposed system, surgeons can operate SILS using their past experience in normal laparoscopic surgery rather than with operations specific to robots and do not have to spend a lot of time practicing. Our master-slave robot is thus not a robot to which humans must adjust but rather "a robot tailored to humans (surgeons)". Even surgeons who have no skill in SILS can perform single-incision laparoscopic surgery by using our proposed system if they have laparoscopic skills. Furthermore, when a surgeon uses our robot system as a one-sided (left-side) instrument, we think that both surgeons and patients can derive a sense of safety from the surgeon touching tissues directly using the other instrument (right-side instrument); in contrast, only the robot directly touches tissues in surgical robot systems like bimanual robots (19) and all-in-one robots (20). Furthermore, our master-slave robot is compact and light, making it usable even in a narrow space. Surgeons are able to respond immediately to emergency situations like sudden bleeding because they operate the system in front of the patient locally. Our proposed locally operated robot system can therefore be expected to reduce the burden of surgeons and make SILS more comfortable for both patients and surgeons.

However, our robot system still has performance problems, and we need to expand the workspace and to improve the positional accuracy and tracking performance of the slave robot to further validate our proposal. In this paper we focused on the robot for supporting single-port laparoscopic cholecystectomy. We plan to expand our proposed system to other surgeries (not only SILS but also conventional multi-port surgery) in our future work. Our proposed locally operated system can increase the number of ports virtually like when a single port is changed to two ports when SILS is employed and enables surgeons to perform in operating areas where they previously could not. In thoracoscopic surgery, the position of the openings is limited due to anatomy, for example surgeons have to insert the surgical instruments 
between costae. When using our robot system in this case, surgeons could decide the appropriate port position for ease of use, and the quality of surgeries should be improved. Hence we need to further improve our robot system according to the objectives of surgeries.

We are planning to add another master-slave robot system for the "right" hand (24) and to evaluate the robot system with in vivo experiments. Surgeons should be able to operate SILS with "the same" surgical techniques as normal multi-port surgery by using our robot system for both hand instruments.

d) Conclusions

We have developed a novel master-slave robot system for the "left" hand that enables surgeons to perform SILS with the operability and visual field similar to regular laparoscopic surgery. In order to evaluate the feasibility and validity of our system, we conducted a basic object-moving task and an ex vivo experiment. We confirmed that the proposed system was useful for SILS, providing a shorter switching time, stable operation, fewer instrument collisions, and reduced stress. Moreover, this study demonstrated the suitability of our proposed system for single-port laparoscopic cholecystectomies. 
Acknowledgments, Disclosure of Interest

- Acknowledgments

This research was supported by a Grant-in-Aid for Japan Society for the Promotion of Science Fellows (A2426910). We are grateful to Mr. Takeharu Kobayashi and Mr. Kohei Kazuhara of Daiken Medical Co. Ltd., who supported the development of the master device.

- Disclosure of Interest

The authors declare that there are no conflicts of interest in this research. 


\section{5) References}

(1) Autorino R, Kaouk JH, Stolzenburg J, Gill IS, Mottrie A, Tewari A, et al. Current Status and Future Directions of Robotic Single-Site Surgery: A Systematic Review. Eur Urol 2013; 63: 266-80.

(2) Ross S, Rosemurgy A, Albrink M, Choung E, Dapri G, Gallagher S, et al. Consensus statement of the consortium for LESS cholecystectomy. Surg Endosc 2012; 26: 2711-6.

(3) Romanelli JR, Earle DB. Single-port laparoscopic surgery: an overview. Surg Endosc 2009; 23: 1419-27.

(4) Mutter D, Callari C, Diana M, Dallemagne B, Leroy J, Marescaux J. Single port laparoscopic cholecystectomy: which technique, which surgeon, for which patient? A study of the implementation in a teaching hospital. J Hepatobiliary Pancreat Sci 2011; 18: 453-7.

(5) Ma J, Cassera MA, Spaun GO, Hammill CW, Hansen PD, Aliabadi-Wahle S. Randomized controlled trial comparing single-port laparoscopic cholecystectomy and four-port laparoscopic cholecystectomy. Ann Surg 2011; 254: 22-7.

(6) Tang B, Hou S, Cuschieri SA. Ergonomics of and technologies for single-port lapaxroscopic surgery. Minim Invasive Ther Allied Technol 2012; 21: 46-54.

(7) White MA, Haber GP, Autorino R, Khanna R, Forest S, Yang B, et al. Robotic Laparoscopic Single-site Radical Prostatectomy: Technique and Early Outcomes. Eur Urol 2010; 58: 544-50.

(8) Erbella J Jr JE, Bunch GM. Single-incision laparoscopic cholecystectomy: the first 100 outpatients. Surg Endsc 2010; 24: 1958-61.

(9) Kroh M, El-Hayek K, Rosenblatt S, Chand B, Escobar P, Kaouk J, et al. First human surgery with a novel single-port robotic system: cholecystectomy using the da Vinci Single-Site platform. Surg Endosc 2011; 25: 3566-73.

(10) Spinoglio G, Lenti LM, Maglione V, Lucido FS, Priora F, Bianchi PP, et al. Single-site robotic cholecystectomy (SSRC) versus sinle-incision laparoscopic cholecystectomy (SILC): comparison of learning curves. First European experience. Surg Endosc 2012; 26: 1648-55.

(11) Pietrabissa A, Sbrana F, Morelli L, Badessi F, Pugliese L, Vinci A, et al. Overcoming the Challenges of Single-Incision Cholecystectomy With Robotic Single-Site Technology. Arch Surg 2012; 147: 709-14.

(12) Field JB, Benoit MF, Dinh TA, Diaz-Arrastia C. Computer-enhanced robotic surgery in gynecologic oncology. Surg Endosc 2007; 21: 244-6.

(13) Weinberg L, Rao S, Escobar PF. Robotic Surgery in Gynecology: An Updated 
Systematic Review. Obstet and Gynecol Int 2011; 2011:852061.

(14) Byrn JC, Schuluender S, Divino CM, Conrad J, Gurland B, Shlasko E, et al. Three-dimensional imaging improves surgical performance for both novice and experienced operators using the da Vinci Robot System. Am J Surg 2007; 193: 519-22.

(15) Kenngott HG, Fischer L, Nickel F, Rom J, Rassweiler J, Müller-Stich BP. Status of robotic assistance - a less traumatic and more accurate minimally invasive surgery?. Langenbecks Arch Surg 2012; 397: 333-41.

(16) Atallah SB, Albert MR, DeBeche-Adams TH, Larach SW. Robotic transanal minimally invasive surgery in a cadaveric. Tech Coloproctol 2011; 15: 461-4.

(17) Turchetti G, Palla I, Pierotti F, Cuschieri A. Economic evaluation of da Vinci-assisted robotic surgery: a systematic review. Surg Endosc 2012; 26: 598-606.

(18) Park S, Kim M, Park MS, Hyung WJ. Rapid adaptaion of robotic gastrectomy for gastric cancer by experienced laparoscopic surgeons. Surg Endosc 2012; 26: 60-7.

(19) Piccigallo M, Scarfogliero U, Quaglia C, Petroni G, Valdastri P, Menciassi A, et al. Design of a novel bimanual robotic system for single port laparoscopy. IEEE ASME Trans Mechatron 2010; 15: 871-8.

(20) Kobayashi Y, Tomono Y, Sekiguchi Y, Watanabe H, Toyoda K, Konishi K, et al. A surgical robot with vision field control for single port endoscopic surgery. Int $\mathbf{J}$ Med Robot 2010; 6: 454-64.

(21) Horise Y, Nishikawa A, Sekimoto M, Kitanaka Y, Miyoshi N, Takiguchi S, et al. Development and evaluation of a master-slave robot system for single-incision laparoscopic surgery. Int J Comput Assist Radiol Surg 2012; 7: 289-96.

(22) Natali CD, Valdastri P. Remote active magnetic actuation for a single-access surgical robotic manipulator. Int J Comput Assist Radiol Surg 2012; 7: S169-76.

(23) Derossis AM, Fried GM, Abrahamowicz M, Sigman HH, Barkun JS, Meakins JL. Development of a Model for Training and Evaluation of Laparoscopic Skills. Am J Surg 1998; 175: 482-7.

(24) Horise Y, Tsumura R, Sekimoto M, Takiguchi S, Doki Y, Mori M, et al. Proposal for a "Patient-mounted" Master-slave Robot System for Single-incision Laparoscopic Surgery. Trans Jpn Soc Med Biol Eng 2013; 51 (supplement): R-263. 